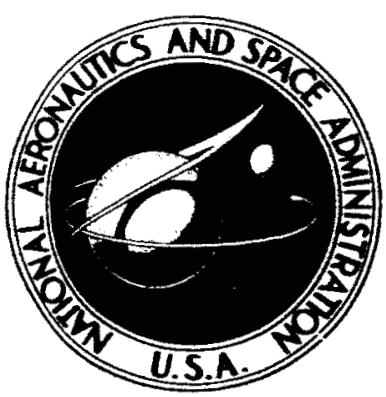

NASA TN D-2806
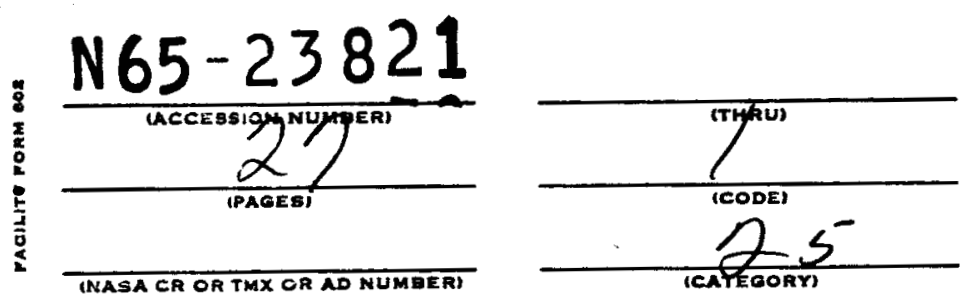

\title{
EQUILIBRIUM COMPUTATIONS FOR MULTICOMPONENT PLASMAS
}

by Frank J. Zeleznik and Sanford Gordon Lewis Research Center Cleveland, Obio

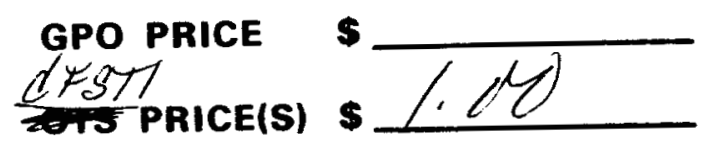

Hard copy $(\mathrm{HC})$

Microfiche (MF)

$+27$

mational aeronautics and SPACE administration - MaShington, D. C. - maY 1965 
By Frank J. Zeleznik and Sanford Gordon

Lewis Research Center

Cleveland, Ohio 


\title{
EQUIIIBRIUM COMPUTATIONS FOR MULTICOMPONENT PLASMAS
}

\author{
by Frank J. Zeleznik and Sanford Gordon
}

Lewis Research Center

\begin{abstract}
SUMMARY
23821

A method is presented for the calculation of multicomponent plasma properties. The method includes both Debye-Huckel and ionization potential lowering effects. These effects are shown to be partially compensating. A perturbation technique is used to make the calculation compatible with a scheme used for calculating ideal gas properties. Thermodynamic derivatives, such as heat capacity, can be calculated directly.
\end{abstract}

\section{INTRODUCTION}

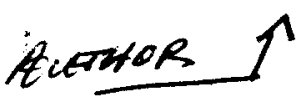

Plasmas have become important in many areas of scientific and technical work. Often the important plasma systems are not simple one-component systems but may involve many different chemical elements. There are several schemes (ref. 1) and many computer programs for the computation of the equilibrium properties of thermodynamic systems that can be regarded as mixtures of ideal gases. These methods, however, must be modified to account for the presence of Coulomb forces.

In principle, the presence of long-range Coulomb forces in a plasma invalidates the independent-particle (ideal gas) approach and requires the consideration of interactions among all particles in the system. Even for simple systems, however, this approach is extremely difficult and has yielded an exact solution only for a one-dimensional plasma model (refs. 2 and 3). A scheme is presented in this report for calculating the thermodynamic properties of many component plasmas that can be applied to most systems and can be expected to yield results which are an improvement over the ideal gas calculations.

The thermodynamic properties for a system of independent particles (ideal gas) can be calculated from a partition function that is a product of two factors. One factor $Q_{t r}$ represents the contribution of the translational kinetic energy to the partition function while the other factor $Q_{\text {int }}$ represents the contributions from the internal energy levels. Because of the assumed independence of the particles, the energy levels used to calculate the internal partition functions are the energy levels for an isolated particle. These energy levels are available for many species from spectroscopic data. Plasma computations that assume ideal gas behavior and neglect Coulomb effects 
are typified by calculations of Kubin and Presley (ref. 4) and early computations of Hilsenrath (ref. 5) and Rouse (refs. 6 and 7). A calculation that assumes nonideal behavior but still neglects Coulomb effects is reported by Rosenbaum and Levitt (ref. 8).

An attempt to incorporate the effect of Coulomb forces produces two changes in the partition function from the partition function in the ideal gas case. First, a third factor appears in the partition function. This factor accounts for the effect of interparticle forces upon the translational partition function and represents the contribution to the partition function of the classical configuration integral. Second, in calculating the internal partition function, one should no longer use the energy levels of an isolated atom or molecule but should use the energy levels of the atom or molecule in the external field produced by the other particles of the system (refs. 9, 10, and 11). Thus, for a plasma, the internal partition function is dependent upon the amount of ionization in the plasma and therefore is a function of the charged particle concentrations. The concentration dependence of the energy levels is generally unknown. Some recent attempts have been made to estimate this effect for hydrogen-like atoms by calculating the energy levels for a screened Coulomb potential (refs. 10 and 12 to 15). No data of this type, however, exist for other species. An alternate and somewhat simpler technique has also been used to take into account the fact that the internal partition function has concentration dependence. This method uses isolated particle energy levels together with an energy level cutoff and an ionization potential lowering that are dependent upon electron concentration; it has been used by Rouse (refs. 16 to 18), Drellishak, Knopp, and Cambel (ref. 19), and Drellishak (ref. 20). The difference between the two procedures, then, is that the first adjusts internal energy levels by varying amounts while the second, in effect, adjust the levels by a constant amount. When only the ground state is used, as by Harris (ref. 2I), the two procedures are equivalent.

The previous discussion was concerned with the effect of Coulomb forces on the internal partition function $Q_{\text {int. }}$. The effect of these forces on the translational partition function $Q_{t r}$ is usually calculated by assuming the validity of the Debye-Hickel approximation. The Debye-Hickel theory is discussed in various textbooks (e.g., refs. 22 and 23 ). Its validity is discussed by Balazs ( $r e f .24$ ) in connection with the one-dimensional plasma for which an exact solution is available. Duclos and Cambel (ref. 25) summarize the conclusions of various authors regarding the region of validity of the Debye-Hickel approximation for three-dimensional plasmas. Myers, Buss, and Benson (ref. 26), Hilsenrath and Klein (ref. 27), Harris (ref. 28), Harris and Trulio (ref. 29), and McGee and Heller (refs. 30 and 31), among others, have alI used the DebyeHückel approximation in calculations. Unfortunately, the calculations of McGee and Heller are in error since they mistakenly use the excess Helmholtz free energy for the excess Gibbs free energy and, in addition, they use an incorrect expression for the excess entropy.

Some confusion exists in the literature regarding the term "ionization potential lowering." This term was discussed by Ecker and Kroll (ref. 32 ), who showed that both the translational and internal energy level effects could be regarded as an ionization potential lowering. This was also mentioned by Harris (ref. 2I). In this report the term "ionization potential lowering" will 
mean only the effects associated with internal energy levels.

The previously discussed Coulomb effects on both $Q_{t r}$ and $Q_{i n t}$ are included in this report. For the effect on $Q_{t r}$, the validity of the Debye-Huckel limiting law approximation is assumed. For the effect on Qint, an ionization potential lowering that is proportional to $k$, the reciprocal of the Debye length, is assumed. This assumption is equivalent to that of Harris (ref. 2l) and Drellishak, Knopp, and Cambel (ref. 19). In performing numerical computations, some scheme must also be used for cutting off the infinite number of unperturbed internal energy levels. This aspect of the problem will not be considered further; however, several of these cutoff schemes are reviewed by Hester and Sewell (ref. 33) and also by Margenau and Lewis (ref. II).

The usual procedure for calculating the thermodynamic properties of plasmas is to assign volume and temperature as the independent thermodynamic parameters. In contrast, the method developed in this report assigns the pressure and one other thermodynamic variable selected from the three quantities temperature, enthalpy, and entropy as independent parameters. For many applications, it is more convenient to have data as functions of pressure rather than volume. In addition, this procedure permits the calculation of thermodynamic derivatives such as specific heat without resorting to numerical differentiations of tabular data (ref. 19).

\section{THERMODYNAMIC FUNCTIONS FOR MULTCOMPONENT PLASMA}

The Helmholtz free energy $A$ of a plasma may be written as

$$
A=A_{I}+A_{C}
$$

where the subscript I denotes the ideal gas contribution and $c$ denotes the contribution from Coulomb forces. (A1I symbols are defined in the appendix.) In terms of the canonical partition function for an ideal gas system,

$$
A_{I}=-k T \ln \left(Q_{t r} Q_{\text {int }}\right)
$$

where $T$ is the absolute temperature and $\mathrm{k}$ is Boltzmann's constant. The translational partition function is given by

$$
Q_{t r}=\prod_{i} \frac{1}{N_{i} !}\left[v\left(\frac{2 \pi \hbar^{2}}{m_{i} k T}\right)^{-3 / 2}\right]^{N_{i}}
$$

where $V$ is the volume, $\hbar$ is Planck's constant divided by $2 \pi, m_{i}$ is the mass per particle of species $i$, and $N_{i}$ is the number of particles of species $i$. The internal partition function is given by

$$
Q_{\text {int }}=\prod_{i}\left[Q_{i n t}^{(i)}\right]^{N_{i}}
$$

where the internal partition function for the $i^{\text {th }}$ species is 


$$
Q_{\text {int }}^{(i)}=e^{-E_{0}^{(i)} / k T} \sum_{i} e^{-\epsilon_{i}^{(i)} / k T}
$$

where $E_{0}^{(i)}$ is the ground state energy for the $i^{\text {th }}$ isolated species and $\epsilon_{i}^{(i)}$ are the isolated energy levels relative to the ground state; $Q_{\text {int }}$ is independent of volume.

The contribution of the Coulomb forces to the Helmholtz free energy can be written in the form

$$
\frac{A_{c}}{k T}=-\frac{1}{12 \pi} V K^{3}+\sum_{i} N_{i} \frac{\Delta E(i)}{k T}
$$

The first term in this equation represents the contribution of the Debye-Hückel limiting law (refs. 22 and 23); that is, it is the effect of coulomb forces on the translational partition function. The second term represents the contribution from the displacement of the internal energy levels. It arises because the truncated partition function $Q_{i n t}^{(i)}$ goes to $Q_{i n t}(i) \exp (-\triangle E(i) / k T)$ when each internal energy level is displaced by the same amount $\Delta E^{(i)}$. The amount of energy level displacement will be a function of ionization. The quantity $k$ is the reciprocal of the Debye length and is given by

$$
k^{2}=\frac{4 \pi}{\mathrm{VkT}} \sum_{i} \mathbb{N}_{i} q_{i}^{2}
$$

where $q_{i}$ is the charge on the $i^{\text {th }}$ species. The dielectric constant that usually appears in the definition of the Debye length has been set equal to unity in equation (7). The displacement of the internal energy levels of the $i_{\text {th }}$ species is assumed to be proportional to $k$, and the proportionality constants $\beta_{i}$ may be different for each species:

$$
\Delta \mathbb{E}^{(i)}=\beta_{i} k a_{0}
$$

where $\beta_{i}$ has the dimensions of energy and $a_{0}$ is the Bohr radius. Combining equations (6) and (8) gives

$$
\frac{A_{c}}{k T}=-\frac{1}{12 \pi} V k^{3}+\frac{k{ }_{0}}{k T} \sum_{i} \beta_{i} N_{i}
$$

The assumption (eq. ( 8$)$ ) is consistent with the assumptions made by Harris (ref. 2l) and Drellishak, Knopp, and Cambel (ref. 19). For the hydrogen plasma, Harris used $\beta_{H}=2 I_{H}$ where $I_{H}$ is the hydrogen atom ionization potential. For species in the argon plasma, Drellishak assumed that the change in ionization potential $\Delta I$ was given by the expression 


$$
\Delta I\left(A r^{+j}\right)=-\frac{I\left(A r^{+j}\right)^{k a_{0}}}{(j+I)} \quad j=0,1,2,3,4
$$

If it is assumed that the energy of the free electron is zero, the ionization potential of successively ionized species of argon can be expressed in terms of their isolated particle ground state energies

$$
I_{\left(\mathrm{Ar}^{+}{ }^{+}\right)}=E_{0}^{(j+1)}-E_{0}^{(j)} \quad j=0,1,2,3,4
$$

Therefore, the change in ionization potential is

$$
\Delta I_{\left(A r^{+j}\right)}=\Delta E(j+1)-\Delta E(j) \quad j=0,1,2,3,4
$$

The use of equations (8), (10), and (11) implies the following $\beta^{\prime}$ s:

$$
\begin{gathered}
\beta_{A r}+j=\sum_{i=j}^{4} \frac{I_{i+1}+i}{i+I} j=0,1,2,3,4 \\
\beta_{\left(\mathrm{Ar}^{+5}\right)}=0
\end{gathered}
$$

Once the form of the Helmholtz free energy (eq. (9)) has been selected, all other thermodynamic functions can be calculated from it by the usual thermodynamic relations. For example, with $U=-T^{2}[\partial(\mathrm{A} / \mathrm{T}) / \partial T]_{V, N_{i}}$ the internal energy can be calculated as

$$
\mathrm{U}=\mathrm{U}_{\mathrm{I}}+\mathrm{U}_{\mathrm{c}}
$$

where

$$
\frac{U_{c}}{k T}=-\frac{1}{8 \pi} V \kappa^{3}+\frac{3}{2} \frac{k a_{0}}{k T} \sum_{i} \beta_{i} N_{i}
$$

The equation of state for the system is obtained from the thermodynamic relation $\mathrm{P}=-(\partial A / \partial V)_{T, N_{i}}$ and can be written in the form

$$
\frac{P V}{k T \sum_{i} N_{i}} \equiv Z=1-\frac{1}{24 \pi} \frac{V K}{\sum_{i} N_{i}}+\frac{1}{2} \frac{k a_{0}}{k T} \frac{\sum_{i} \beta_{i} N_{i}}{\sum_{i} N_{i}}
$$

The deviation of $\mathrm{Z}$ from unity indicates the departure from ideal behavior due to Coulomb forces, and thus $\mathrm{Z}$ will be called the Coulomb compressibility. The two terms in equation (15) that account for the deviation from unity are compensating if the $\beta_{i}$ are taken to be positive. In terms of $z$, equations (9) and (14) become 


$$
\begin{aligned}
& \frac{A_{c}}{k T}=2(Z-1) \sum_{i} N_{i} \\
& \frac{U_{c}}{k T}=3(Z-1) \sum_{i} N_{i}
\end{aligned}
$$

The enthalpy $\mathrm{H}$ and the ideal gas enthalpy $\mathrm{H}_{\mathrm{I}}$ are defined as

$$
\begin{gathered}
H=U+P V=U_{I}+U_{C}+P V \\
H_{I}=U_{I}+k T \sum_{i} N_{i}
\end{gathered}
$$

Equations (15), (17), (18), and (19) may be combined to give

$$
\mathrm{H}=\mathrm{H}_{\mathrm{I}}+4(\mathrm{Z}-\mathrm{I}) \mathrm{kT} \sum_{\mathrm{i}} \mathrm{N}_{\mathrm{i}}
$$

The expression for entropy in terms of volume and temperature can be obtained from the thermodynamic relation $S=-(\partial \mathrm{A} / \partial \mathrm{T})_{\mathrm{V}, \mathrm{N}_{i}}$. Just as in the case of the other thermodynamic functions, the contribution of the Coulomb forces can be written in terms of the Coulomb compressibility:

$$
S=S_{I}+(Z-I+\ln Z) k \sum_{i} N_{i}
$$

The In $\mathrm{Z}$ term in the correction for nonideality arises because the volume was eliminated by means of the equations of state (15). Usually in $\mathrm{Z}$ is ignored; however, by doing this one is disregarding a term that is of the same order of magnitude as the term retained.

Expressing the equations for enthalpy and entropy in terms of moles $n_{i}$ rather than the number of particles $N_{i}$ gives

$$
\begin{gathered}
\mathrm{H}=\sum_{i=1}^{\mathrm{m}}\left(\mathrm{H}_{\mathrm{T}}^{\mathrm{O}}\right)_{i} \mathrm{n}_{i}+4(\mathrm{z}-1) \mathrm{RT} \sum_{i=1}^{\mathrm{m}} \mathrm{n}_{i} \\
\mathrm{~S}=\sum_{i=1}^{\mathrm{m}}\left(\mathrm{ST}_{\mathrm{T}}\right)_{i} \mathrm{n}_{i}+(\mathrm{z}-1+\ln \mathrm{Z}) \mathrm{R} \sum_{i=1}^{\mathrm{m}} \mathrm{n}_{i}
\end{gathered}
$$

where

$$
\left(S_{T}\right)_{i}=\left(S_{T}^{\circ}\right)_{i}-R \ln p_{i}
$$




$$
p_{j}=\frac{n_{j} P}{\sum_{i=1}^{m} n_{i}}
$$

In these equations it has been assumed that there are $m$ different species in the system. With the use of equations (22) and (23) the Gibbs free energy becomes

$$
F=F_{I}+F_{c}
$$

where

$$
\begin{gathered}
F_{I}=\sum_{i=1}^{m} n_{i}\left[\left(H_{T}^{O}\right)_{i}-T\left(S_{T}\right)_{i}\right] \\
F_{c}=[3(z-1)-\ln z] R T \sum_{i=1}^{m} n_{i}
\end{gathered}
$$

\section{INVERSION OF EQUATION OF STATE}

It is desired to express the equations of this report in terms of pressure, rather than in terms of volume, as one of the independent parameters. Thus an expression for volume in terms of pressure is necessary in order to eliminate volume from all thermodynamic functions. This expression can be obtained by inverting the equation of state. This inversion is equivalent to expressing the Coulomb compressibility in terms of pressure and temperature.

If the dimensionless ionization parameter

$$
\alpha \equiv-\frac{1}{3}\left(\frac{\pi P}{k T}\right)^{1 / 2}\left(\frac{\sum_{i} N_{i} q_{i}^{2}}{k T \sum_{i} N_{i}}\right)^{3 / 2}\left(1-3 a_{0} \frac{\sum_{i} \beta_{i} N_{i}}{\sum_{i} N_{i} q_{i}^{2}}\right)
$$

is introduced, the equation of state (15) becomes

$$
1+\alpha z^{-1 / 2}=z
$$

The analytical solution of equation (28) in the form $Z=Z(\alpha)$ can be found conveniently by studying the associated cubic equation

$$
z^{3}-2 z^{2}+z-a^{2}=0
$$

This equation was obtained from equation (28) by rearranging terms and then squaring. Not all roots of the cubic equation (29) are valid solutions for the equation of state (28). The solution that is valid for both equations is 


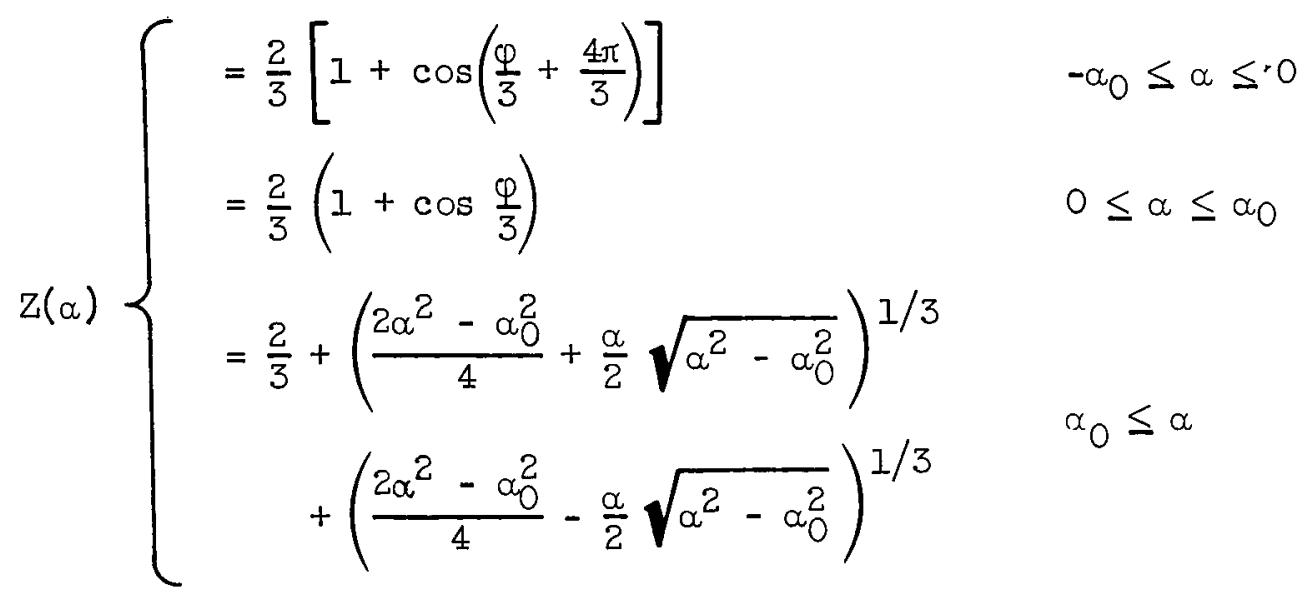

where

$$
\cos \varphi=-1+\frac{2 \alpha^{2}}{\alpha_{0}^{2}}
$$

where

$$
\alpha_{0}=\frac{2}{3 \sqrt{3}}
$$

Figure $I$ is a plot of $z$ as a function of $\alpha$. If the ionizaticn potential lowering is neglected, $\alpha \leq 0$ and $Z<I$; whereas, if the Debye-Huickel limiting law is neglected, $\alpha \geq 0$ and $z>1$. Because these two contributions are partially compensating, considerable ionization can occur with little effect on the equation of state. It may be noted that there is no solution for $\alpha<-\alpha_{0}$. At $\alpha=-\alpha_{0}, Z$ has the value $1 / 3$ while, at $\alpha=\alpha_{0}, Z$ equals $4 / 3$.

For $|\alpha| \leq 0.1$, the trigonometric solution (eq. (30)! can be replaced by the expansion

$$
z \cong 1+\alpha-\frac{\alpha^{2}}{2}+\frac{5}{8} \alpha^{3}-\alpha^{4}+\frac{231}{128} \alpha^{5} \quad|\alpha| \leq 0.1
$$

This expansion gives at least seven figure accuracy in this interval.

\section{ITERATION EQUATIONS}

In this section, the iteration equations used to walculate chemical equilibrium will be derived. In the following section, the equations required for the calculation of thermodynamic derivatives will be given. For the purposes of this report, the derivations will be based on the minimization of the Gibbs free energy and will be related to the somewhat different free energy minimization methods described by White, Johnson, and Dantzig (ref. 34) and Zeleznik and Gordon (ref. 1). In order to make the method compatible with the techniques used in the computer programs described by Gordon and Zeleznik 


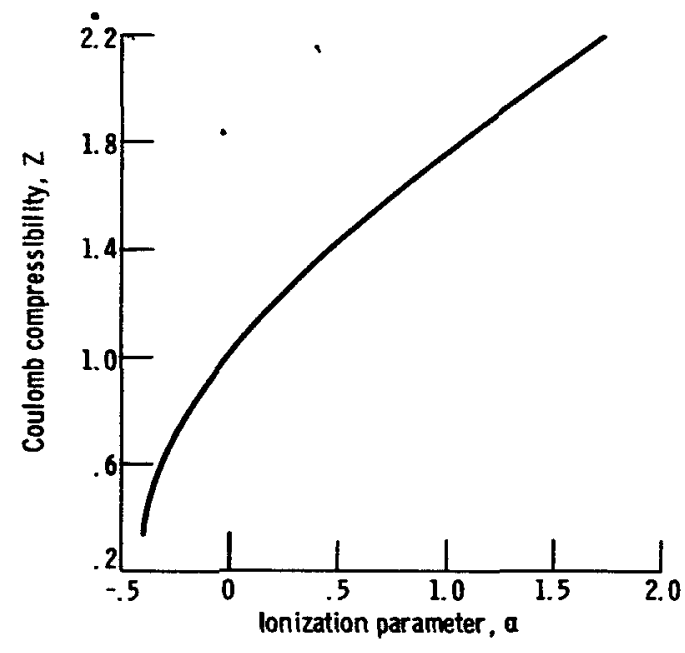

Figure 1. - Coulomb compressibility as function of ionization parameter. (refs. 35 and 36), the derivation used here will be such that in the ideal gas limit the equations will be identical in form to those obtained by the equilibrium constant method (ref. 1). This can be accomplished by regarding the sum of the moles of all gaseous species as fixed at a value that is numerically equal to the assigned pressure $\mathrm{P}_{\mathrm{O}}$. This assumption requires treating the total mass of reactants as a variable just as has been done in the past for the ideal gas (refs. 1 and 37). Since the derivation of the iteration equations closely parallels the minimization procedure of reference 1, many of the details will be omitted.

The thermodynamic state is assumed to be specified by assigning the pressure and one other thermodynamic variable selected from the three quantities, temperature, enthalpy, and entropy. The pressure of the system is specified by

$$
P_{0}-P \equiv \Delta P=0
$$

where $P=\sum_{i=1}^{m} p_{i}$. The specification of the thermodynamic state is completed by assigning either temperature, enthalpy, or entropy as follows:

$$
\begin{gathered}
\mathrm{T}_{0}-\mathrm{T}=0 \\
\mathrm{~h}_{0}-\mathrm{h}=\Delta \mathrm{h}=0 \\
\mathrm{~s}_{0}-\mathrm{s}=\Delta \mathbf{s}=0
\end{gathered}
$$

where

$$
\begin{gathered}
\overline{\mathrm{A} h}=\sum_{i=1}^{m}\left(\mathrm{H}_{\mathrm{T}}^{\mathrm{O}}\right)_{i} \mathrm{n}_{i}+4(\mathrm{z}-\mathrm{I}) \mathrm{RT} \sum_{i=1}^{\mathrm{m}} \mathrm{n}_{\mathrm{i}} \\
\overline{\mathrm{A}} \mathrm{s}=\sum_{i=1}^{\mathrm{m}}\left(\mathrm{S}_{\mathrm{T}}\right)_{i} \mathrm{n}_{\mathrm{i}}+(\mathrm{z}-1+\ln \mathrm{z}) \mathrm{R} \sum_{i=1}^{\mathrm{m}} \mathrm{n}_{\mathrm{i}}
\end{gathered}
$$

The quantity $\bar{A}$ is the total mass of the system. Since the size of the system is arbitrary, it is convenient to let $\bar{A}$ be that mass which is required in order that the partial pressure of each species is numerically equal to the number of moles of that species; that is, $p_{i}=n_{i}$. In the following equations, $n_{i}$ is substituted for $p_{i}$. The value of $\bar{A}$ that permits this substitution is determined simultaneously with the other variables of the problem.

Once the thermodynamic state has been specified, the equilibrium composi- 
tions can be obtained by minimizing the Gibbs free energy subject, to mass. balance constraints. If there are ( 2 - 1) different chemical elements (where $l$ is some fixed integer), the $l$ equations for conservation of species and charge neutrality can be written as

$$
b_{i}^{0}-b_{i} \equiv \Delta b_{i}=0 \quad(i=1 \cdot \cdot \cdot \tau)
$$

where $\overline{\mathrm{A}} \mathrm{b}_{i}=\sum_{j=1}^{m} a_{i, j} n_{j}$. The $a_{i j}$ are the stoichiometric coefficients. In the set of equations ( 38 ), the equation corresponding to $i=2$ refers to the conservation of charge. For the case of a neutral plasma, $b_{i}=0$.

If the state is specified by assigning a temperature and a pressure, the iteration equations are obtained by minimizing the quadratic approximation to F/RT subject to the constraints (eqs. (32) and (33)) and the Iinearized form of equation (38). The mass balance constraint is incorporated by means of Lagrange multipliers $\pi_{i}$, whereas the pressure equation and the temperature equation are used directly. The resulting iteration equations are

$$
\begin{gathered}
\sum_{j=1}^{m}\left(\delta_{k j}+\Gamma_{k j}\right) \Delta \ln n_{j}-\sum_{i=1}^{l} \pi_{i} a_{i k} \\
-\left[\frac{\partial\left(\frac{H}{R T}\right)}{\partial n_{k}}\right]_{T, P} \Delta \ln T=-\left[\frac{\partial\left(\frac{F}{R T}\right)}{\partial n_{k}}\right]_{T, P} \quad(k=I, 2 \cdot . \cdot m) \\
\sum_{j=1}^{m} a_{i j} n_{j} \Delta \ln n_{j}-\bar{A} b_{i} \Delta \ln \bar{A}=\bar{A} \Delta b_{i} \\
\sum_{j=1}^{m} n_{j} \Delta \ln n_{j}=\Delta P
\end{gathered}
$$

where

$$
\begin{aligned}
& n_{j}\left[\frac{\partial^{2}\left(\frac{F_{I}}{R T}\right)}{\partial n_{k} \partial n_{j}}\right]_{P, T}=\delta_{k j} \\
& n_{j}\left[\frac{\partial^{2}\left(\frac{F_{c}}{R T}\right)}{\partial n_{k} \partial n_{j}}\right]_{P, T}=\Gamma_{k j}
\end{aligned}
$$


It may be noted that, for assigned temperature, equation (42) is trivial and may be omitted together with the $\Delta$ In $T$ term in equation (39). If, however, the temperature is specified indirectly by means of equation (34), then the linearized form of equation (34) is used in place of equation (42):

$$
\sum_{j=1}^{m} n_{j}\left[\frac{\partial\left(\frac{H}{R T}\right)}{\partial n_{j}}\right]_{T, P} \Delta \ln n_{j}-\frac{H}{R T} \Delta \ln \bar{A}+\left[\frac{\partial\left(\frac{H}{R}\right)}{\partial T}\right]_{P, n_{j}} \Delta \ln T=\frac{\bar{A} \Delta h}{\overline{R T}}
$$

The following equation, (45), is used when the temperature is specified indirectly by means of equation (35) instead of equation (42). This equation is the linearized form of equation (35) to which has been added equation (4I) multiplied by the factor $(2-z-\ln z)$ :

$$
\begin{aligned}
& \sum_{j=1}^{m}\left\{n_{j}\left[\frac{\partial\left(\frac{S}{R}\right)}{\partial n_{j}}\right]_{T, P}+n_{j}(2-z-\ln z)\right\} \Delta \ln n_{j}-\frac{S}{R} \Delta \operatorname{In} \bar{A} \\
& +T\left[\frac{\partial\left(\frac{S}{R}\right)}{\partial T}\right]_{P, n_{j}} \Delta \ln T=\frac{\bar{A} \Delta s}{R}+\Delta P(2-z-\text { In } z)
\end{aligned}
$$

The most important difference between the preceding sets of iteration equations and the corresponding equations for the case of an ideal gas is the appearance of the matrix $\Gamma$ in equation (39). In the ideal case $(\Gamma=0)$, these equations could be used to eliminate $\Delta$ In $n_{j}(j=I .$. m) in terms of $\pi_{i}(i=1 . . . \imath), \Delta$ In $\bar{A}$, and $\Delta$ In $T$. Thus the number of equations could be reduced from $m+z+2$ to $l+2$ and the amount of numerical computation

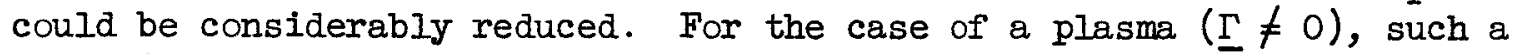
reduction cannot be performed as easily. This problem will be discussed more fully in a later section.

\section{THERMODYNAMIC FIRST DERIVATIVES}

In addition to the thermodynamic functions themselves, it is often desirable to have available the first derivatives of the thermodynamic functions. All possible first derivatives can be expressed in terms of any three independent ones. As in earlier papers (refs. 35 and 37), the heat capacity at constant pressure 


$$
c_{p} \equiv\left(\frac{\partial H}{\partial T}\right)_{P}=\left(\frac{\partial H}{\partial T}\right)_{P, n_{i}}+\sum_{i=1}^{m} \frac{n_{i}}{T}\left(\frac{\partial H}{\partial n_{i}}\right)_{T, P}\left(\frac{\partial \ln n_{i}}{\partial \ln T}\right)_{P}
$$

and the derivatives of volume with respect to temperature and pressure ( $\partial \mathrm{V} / \partial \mathrm{T})_{P}$ and $(\partial \mathrm{V} / \partial \mathrm{P})_{\mathrm{T}}$ are selected as the independent derivatives. If an average molecular weight is introduced, the equations of state can be written

$$
\mathrm{PV}=\mathrm{z} \frac{\mathrm{W}}{\mathrm{M}} \mathrm{RT}
$$

This equation leads to the relations

$$
\begin{gathered}
\left(\frac{\partial \ln V}{\partial \ln T}\right)_{P}=1-\left(\frac{\partial \ln M}{\partial \ln T}\right)_{P}+\frac{1}{Z} \frac{d z}{d x}\left[\left(\frac{\partial \alpha}{\partial \ln T}\right)_{P, n_{i}}\right. \\
\left.+\sum_{i=1}^{m} n_{i}\left(\frac{\partial \alpha}{\partial n_{i}}\right)_{T, P}\left(\frac{\partial \ln n_{i}}{\partial \ln T}\right)_{P}\right] \\
\left(\frac{\partial \ln V}{\partial \ln P}\right)_{T}=-\left[1+\left(\frac{\partial \ln M}{\partial \ln P}\right)_{T}\right]+\frac{1}{Z} \frac{d Z}{d \alpha}\left[\left(\frac{\partial \alpha}{\partial \ln P}\right)_{T, n_{i}}{ }^{m} \sum_{i=1}^{m} n_{i}\left(\frac{\partial \alpha}{\partial n_{i}}\right)_{T, P}\left(\frac{\partial \ln n_{i}}{\partial \ln P}\right)_{T}\right]
\end{gathered}
$$

From the procedure of reference (37), it is readily established that

$$
\begin{gathered}
\left(\frac{\partial \ln M}{\partial \ln T}\right)_{P}=\left(\frac{\partial \ln \bar{A}}{\partial \ln T}\right)_{P} \\
1+\left(\frac{\partial \ln M}{\partial \ln \bar{P}}\right)_{T}=\frac{P}{\sum_{i=1}^{m} n_{i}\left(\frac{\partial \ln n_{i}}{\partial \ln \bar{A}}\right)_{T}} \\
\left(\frac{\partial \ln n_{i}}{\partial \ln P}\right)_{T}=\frac{P\left(\frac{\partial \ln n_{i}}{\partial \ln \bar{A}}\right)_{T}}{\sum_{k=1}^{m} n_{k}\left(\frac{\partial \ln n_{k}}{\partial \ln \bar{A}}\right)_{T}}
\end{gathered}
$$


Therefore, the evaluation of the three independent first derivatives reduces to the problem of calculating derivatives with respect to In $T$ at constant pressure and with respect to in $\bar{A}$ at constant temperature. The derivatives with respect to In $T$ are obtained by solving the set of equations

$\sum_{j=1}^{m} n_{j} \frac{\partial U_{k}}{\partial n_{j}}\left(\frac{\partial \ln n_{j}}{\partial \ln T}\right)_{P}+\sum_{i=1}^{l} \frac{\partial U_{k}}{\partial \pi_{i}}\left(\frac{\partial \pi_{i}}{\partial \ln T}\right)_{P}+\left(\frac{\partial U_{k}}{\partial \ln T}\right)_{P}=0$

$$
(\mathrm{k}=1,2,3 \cdot \ldots \mathrm{m})
$$

$\sum_{j=1}^{m}$

$$
a_{i j} n_{j}\left(\frac{\partial \ln n_{j}}{\partial \ln T}\right)_{P}-\overline{A b}_{i}^{o}\left(\frac{\partial \ln \bar{A}}{\partial \ln T}\right)_{P}=0 \quad(i=1,2,3 . . .2)
$$

$$
\sum_{j=1}^{m} n_{j}\left(\frac{\partial \ln n_{j}}{\partial \ln T}\right)_{P}=0
$$

where

$$
U_{k} \equiv \frac{\partial}{\partial n_{k}}\left[\frac{F}{R T}+\sum_{i=1}^{l} \pi_{i}\left(\bar{A}_{i}^{0}-\sum_{j=1}^{m} a_{i j} n_{j}\right)\right]_{T, P}=\left[\frac{\partial\left(\frac{F}{R T}\right)}{\partial n_{k}}\right]_{T, P}-\sum_{i=1}^{l} \pi_{i} a_{i k}
$$

These $m+2+1$ equations are identical in form to the $m+2+1$ iteration equations (39) to (4I) although they are not derived from the iteration equations. They can be thought of, however, as being obtained from the iteration equations by setting the right-hand side equal to zero and by formally dividing through by $\Delta$ In $T$.

The derivatives with respect to In $\bar{A}$ are obtained by solving the set of equations

$$
\begin{gathered}
\sum_{j=1}^{m} n_{j} \frac{\partial u_{k}}{\partial n_{j}}\left(\frac{\partial \ln n_{j}}{\partial \ln \bar{A}}\right)_{T}+\sum_{i=1}^{l} \frac{\partial u_{k}}{\partial \pi_{i}}\left(\frac{\partial \pi_{i}}{\partial \ln \bar{A}}\right)_{T}=0 \\
(k=1,2,3 \ldots . m) \\
\sum_{j=1}^{m} a_{i j} n_{j}\left(\frac{\partial \ln n_{j}}{\partial \ln \bar{A}}\right)_{T}-\bar{A}_{i}^{O}=0 \\
(i=1,2,3 \ldots . . i)
\end{gathered}
$$


Again there is an obvious similarity between these equations and the iteration equations (39) and (40).

\section{SOLUTION OF EQUATIONS BY PERTURBATION}

The problem of calculating the thermodynamic properties of a multicomponent plasma has been reduced to the problem of solving one of three different sets of simultaneous linear iteration equations. The set of equations (39), (40), (41), (42) is used if the thermodynamic state is specified by assigning a temperature and a pressure. A second set (eqs. (39), (40), (41), (44)) is used if the state is specified by enthalpy and pressure while the third set (eqs. (39), (40), (41), (45)) is used when the state is specified by entropy and pressure. When the iteration procedure has converged, two additional sets of linear equations must be solved in order to calculate the three independent derivatives discussed earlier. One of these sets is composed of equations (53), (54), (55) while the other is formed from equations (57), (58).

These five sets of linear equations are very similar to each other, and therefore simultaneous discussion of the solution of these sets is possible. In the discussion of these equations, the more concise matrix notation $\mathrm{M} \mathrm{v}=\mathrm{w}$ will be used to symbolize any one of the five sets of linear equations. $\bar{F}$ urther the matrix $M$ and the column vectors $\underline{v}$ and $w$ will be assumed to have been partitione $\bar{d}$ so that the set of equations can $\bar{b} e$ written in the form

$$
\left(\begin{array}{ll}
\underline{M}_{11} & -M_{2} \\
\underline{M}_{21} & \underline{M}_{22}
\end{array}\right)\left(\begin{array}{l}
\underline{v}_{1} \\
\underline{v}_{2}
\end{array}\right)=\left(\begin{array}{l}
\underline{w}_{1} \\
\underline{w}_{2}
\end{array}\right)
$$

The column vector $v_{I}$ is associated with the variables related to $\mathrm{n}_{j}(j=1,2, \cdot . \cdot, \mathrm{m})$, while $\mathrm{v}_{2}$ is associated with the remaining variables. The matrix $\underline{M}_{-1}$ is square and has the dimension $m$. The matrix $\underline{M}_{-22}$ is also square and has the dimensions $\imath+2$ for the iteration equations, while for the sets (53), (54), (55) and (57), (58) it has the dimensions $l+1$ and $l$, respectively.

If the set of equations represented by equation (59) is relatively small, one can work directly with these equations. In multicomponent systems, however, the number of equations could be quite large and their solutions could involve a considerable amount of numerical difficulty. For this reason, working with a reduced set of equations analogous to those used for the ideal gas problem would be convenient. As has already been pointed out, the principal difference between equation (59) and the corresponding ideal gas equations lies in the fact in the ideal gas case $M_{11}$ was a unit matrix. This permitted the first member of equation (59) to be used in the direct elimination of $v_{1}$ from the second member of the set and thereby gave a considerably reduced set of linear equations in the variables $\underline{v}_{2}$. Since the objective is to provide a calculating scheme compatible with the ideal gas case, a reduced set of linear equations 
must first be'constructed. With the square submatrix $M I$ assumed to be nonsingular

$$
\underline{v}_{1}=\frac{M^{-1}}{-1}\left(\underline{w}_{-1}-\frac{M}{-12}-\frac{v}{2}\right)
$$

This equation is used to eliminate $\mathrm{v}_{-1}$ from the second member of equation (59), which yields

$$
\left(\underline{M}_{22}-\underline{M}_{21} M_{-1}^{-1}-12\right) \underline{v}_{2}=\underline{w}_{2}-\underline{M}_{2 I} M_{-1}^{-1} w_{-1}
$$

Equations (61) possess the disadvantage that they contain the matrix $M_{-1}^{-1}=(\underline{I}+\underline{\Gamma})^{-1}$. The need for numerically calculating $M_{-1}^{-1}$ would be eliminated if a closed-form expression could be obtained for $\mathrm{M}_{-1}^{-1}$. Obtaining this expression is certainly not possible in a general case, but it can be done when the contribution of $\underline{\Gamma}$ to $\frac{M}{11}$ can be regarded as a perturbation. Then the iterative expansion of $\underline{M}_{-11}^{-1}$ is

$$
\underline{M}_{-1}^{-1}=\sum_{k=0}^{\infty}(-1)^{k} \underline{T}^{k}
$$

where $\underline{\Gamma}^{\circ} \equiv \underline{I}$.

If the notation $\underline{N}_{-}=\underline{y}$ is used for the reduced set of equations (61) and if equation (62) is used to eliminate $\underline{M}_{-1}^{-1}$ from both $N$ and $y$,

$$
\left.\begin{array}{l}
\underline{N}=\sum_{k=0}^{\infty} \underline{N}^{(k)} \\
\underline{y}=\sum_{k=0}^{\infty} \underline{y}^{(k)}
\end{array}\right\}
$$

where

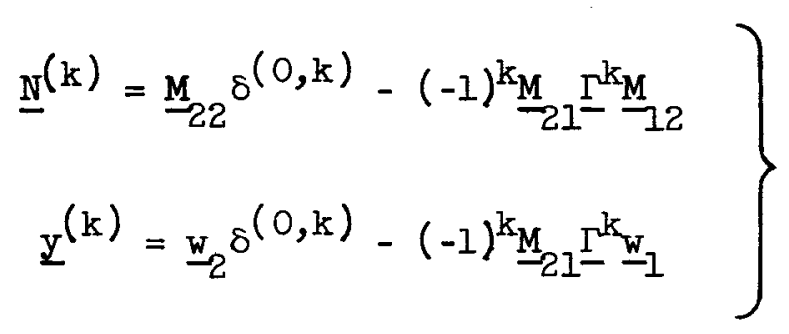

The index $\mathrm{k}$ effectively gives the order to which the perturbation matrix $\underline{\Gamma}$ appears in the various terms. Therefore, conventional perturbation theory can now be applied in the solution of the reduced set of equations (61). Writing 


$$
\underline{\mathrm{v}}_{2}=\sum_{\mathrm{k}=0}^{\infty} \underline{\mathrm{v}}_{2}^{(\mathrm{k})}
$$

and equating terms in equations (61) with the same order of perturbation yields the usual equations

$$
\begin{aligned}
& \underline{\mathrm{N}}^{(0)} \underline{\mathrm{V}}_{2}^{(0)}=\underline{\mathrm{y}}^{(0)} \quad \mathrm{k}=0 \\
& \left.\underline{N}^{(0)} \underline{v}_{2}^{(k)}=y^{(k)}-\sum_{j=1}^{k} \underline{N}^{(j)} \underline{v}_{2}^{(k-j)} \quad k=1,2,3 \ldots\right\}
\end{aligned}
$$

These equations can be used to calculate $\underline{v}_{2}$ to the desired order of perturbation. An expression for $\underline{v}_{-1}$ can then be obtained from $\underline{v}_{2}$ by using equation (60). Grouping terms with the same order gives

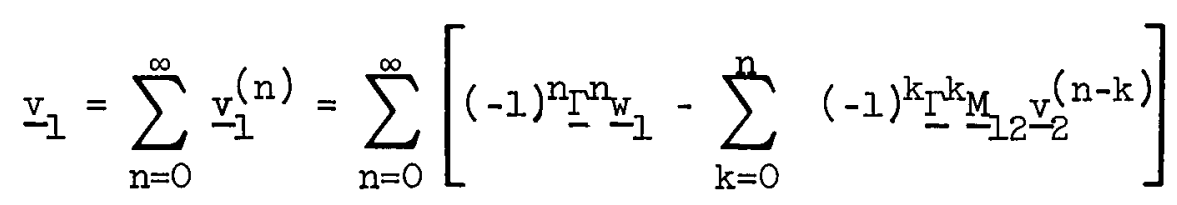

The explicit expressions for the matrices $\mathbb{N}^{(k)}$ and the vectors $y^{(k)}$ be given for the case in which the equations to $\bar{b}$ e solved by perturbation correspond to the iteration equations. Using the notation

$$
\begin{aligned}
& \begin{array}{c}
\alpha_{k} \equiv\left(\frac{\partial \alpha}{\partial n_{k}}\right)_{T, P}=\alpha\left(\frac{1}{2} \frac{q_{k}^{2}}{\sum_{i=1}^{m} n_{i} q_{i}^{2}}-\frac{3}{2} \frac{1}{\sum_{i=1}^{m} n_{i}}+\frac{q_{k}^{2}-3 a_{0} \beta_{k}}{\sum_{i=1}^{m} n_{i} q_{i}^{2}-3 a_{0} \sum_{i=1}^{m} \beta_{i} n_{i}}\right) \\
\alpha_{T} \equiv\left(\frac{\partial \alpha}{\partial \ln T}\right)_{P, n_{i}}=-2 \alpha \\
\alpha_{P} \equiv\left(\frac{\partial \alpha}{\partial \ln P}\right)_{T, n_{i}}=\frac{\alpha}{2}
\end{array} \\
& \begin{array}{c}
\alpha_{k} \equiv\left(\frac{\partial \alpha}{\partial n_{k}}\right)_{T, P}=\alpha\left(\frac{1}{2} \frac{q_{k}^{2}}{\sum_{i=1}^{m} n_{i} q_{i}^{2}}-\frac{3}{2} \frac{1}{\sum_{i=1}^{m} n_{i}}+\frac{q_{k}^{2}-3 a_{0} \beta_{k}}{\sum_{i=1}^{m} n_{i} q_{i}^{2}-3 a_{0} \sum_{i=1}^{m} \beta_{i} n_{i}}\right) \\
\alpha_{T} \equiv\left(\frac{\partial \alpha}{\partial \ln T}\right)_{P, n_{i}}=-2 \alpha \\
\alpha_{P} \equiv\left(\frac{\partial \alpha}{\partial \ln P}\right)_{T, n_{i}}=\frac{\alpha}{2}
\end{array} \\
& \begin{array}{c}
\alpha_{k} \equiv\left(\frac{\partial \alpha}{\partial n_{k}}\right)_{T, P}=\alpha\left(\frac{1}{2} \frac{q_{k}^{2}}{\sum_{i=1}^{m} n_{i} q_{i}^{2}}-\frac{3}{2} \frac{1}{\sum_{i=1}^{m} n_{i}}+\frac{q_{k}^{2}-3 a_{0} \beta_{k}}{\sum_{i=1}^{m} n_{i} q_{i}^{2}-3 a_{0} \sum_{i=1}^{m} \beta_{i} n_{i}}\right) \\
\alpha_{T} \equiv\left(\frac{\partial \alpha}{\partial \ln T}\right)_{P, n_{i}}=-2 \alpha \\
\alpha_{P} \equiv\left(\frac{\partial \alpha}{\partial \ln P}\right)_{T, n_{i}}=\frac{\alpha}{2}
\end{array} \\
& f_{k} \equiv\left[\frac{\partial\left(\frac{F}{R T}\right)}{\partial n_{k}}\right]_{T, P}=\frac{\left(F_{T}^{\circ}\right)_{k}}{R T}+\ln n_{k}+1+3(z-1)-\ln z \\
& +\frac{(3 z-1)}{z} \sum_{i=1}^{m} n_{i} \frac{d z}{d a} \alpha_{k}
\end{aligned}
$$

will 


$$
\begin{gathered}
h_{k} \equiv\left[\frac{\partial\left(\frac{H}{R T}\right)}{\partial n_{k}}\right]_{T, P}=\frac{\left(H_{T}^{\circ}\right)_{k}}{R T}+4(z-I)+4 \sum_{i=1}^{m} n_{i} \frac{d z}{d a} \alpha_{k} \\
h_{T} \equiv\left[\frac{\partial\left(\frac{H}{R}\right)}{\partial T}\right]_{P, n_{i}}=\sum_{i=1}^{m} \frac{\left(c_{p}^{\circ}\right)_{i} n_{i}}{R}+4(z-1) \sum_{i=1}^{m} n_{i}+4 \sum_{i=1}^{m} n_{i} \frac{d z}{d a} \alpha_{T}
\end{gathered}
$$

gives the following forms for $\mathrm{y}^{(\mathrm{k})}$ and $\mathrm{N}^{(\mathrm{k})}$ :

$\underline{N}^{(0)}=\left(\begin{array}{ccc}\sum_{j=1}^{m} a_{i j} a_{k j} n_{j} & \bar{A} b_{i} & \sum_{j=1}^{m} a_{i j} h_{j} n_{j} \\ \sum_{j=1}^{m} a_{k j} n_{j} & 0 & \sum_{j=1}^{m} h_{j} n_{j} \\ \sum_{j=1}^{m} a_{k j} h_{j} n_{j} & \text { H/RT } & h_{T}+\sum_{j=1}^{m} h_{j} h_{j} n_{j}\end{array}\right) i, k=1 \cdot \cdots \cdot$

$$
y(0)=\left(\begin{array}{c}
\bar{A} \Delta b_{i}+\sum_{j=1}^{m} a_{i j} n_{j} f_{j} \\
\Delta+\sum_{j=1}^{m} n_{j} f_{j} \\
\bar{A} \frac{\Delta h}{R T}+\sum_{j=1}^{m} h_{j} n_{j} f_{j}
\end{array}\right) \quad i, k=1 \cdots \cdot
$$

$$
\underline{N}^{(r)}=(-1)^{r}\left(\begin{array}{lll}
\sum_{j, t=1}^{m} a_{i j} n_{j}\left(\underline{\Gamma}^{r}\right)_{j t} a_{k t} & 0 & \sum_{j, t=1}^{m} a_{i j} n_{j}\left(\underline{\Gamma}^{r}\right)_{j t} h_{t} \\
\sum_{j, t=1}^{m} n_{j}\left(\underline{\Gamma}^{r}\right)_{j t} a_{k t} & 0 & \sum_{j, t=1}^{m} n_{j}\left(\underline{\Gamma}^{r}\right)_{j t} h_{t} \\
\sum_{j, t=1}^{m} h_{j} n_{j}\left(\underline{\Gamma}^{r}\right)_{j t} a_{k t} & 0 & \sum_{j, t=1}^{m} h_{j} n_{j}\left(\underline{\Gamma}^{r}\right)_{j t} h_{t}
\end{array}\right)
$$




$$
y^{(r)}=(-1)^{r}\left(\begin{array}{l}
\sum_{j, t=1}^{m} a_{i j} n_{j}\left(\underline{\Gamma}^{r}\right)_{j t} f_{t} \\
\sum_{j, t=1}^{m} n_{j}\left(\underline{\Gamma}^{r}\right)_{j t} f_{t} \\
\sum_{j, t=1}^{m} n_{j} n_{j}\left(\underline{\Gamma}^{r}\right)_{j t} f_{t}
\end{array}\right) \quad . r=1,2,3 \cdots \cdot \cdot
$$

A considerable amount of cancellation occurs in $\underline{N}^{(r)}$ and $\mathrm{y}^{(r)}$ $(r=1,2,3 .$. . ) because of two properties of the matrix $\underline{\Gamma}$.

$$
\begin{aligned}
& \sum_{i=1}^{m} n_{i} \Gamma_{i k}=0 \\
& \sum_{i=1}^{m} \Gamma_{k i}=0
\end{aligned}
$$

These properties are a direct result of Buler's theorem for homogeneous functions. The function $F_{c}$, defined by equation (26), is homogeneous of degree 1 in the $n_{i}$ because $\alpha$ is homogeneous of degree zero in $n_{i}$ and thus so is Z. By Euler's theorem

$$
\sum_{i=1}^{m} n_{i} \frac{\partial F_{c}}{\partial n_{i}}=F_{c}
$$

Differentiation with respect to $\mathrm{n}_{\mathrm{k}}$ gives

$$
\sum_{i=1}^{m} n_{i} \frac{\partial^{2} F_{c}}{\partial n_{k} \partial n_{i}}=0
$$

Equation (8I) gives immediately the second of the properties of $\Gamma$ (eq. (79)). The first property (eq. (78)) also follows from this result by recalling that the order of differentiation is immaterial; and thus for $n_{k}$ not equal to zero equation (81) can be written in the form

$$
\frac{1}{n_{k}} \sum_{i=1}^{m} n_{i} n_{k} \frac{\partial^{2} F_{c}}{\partial n_{i} \partial n_{k}}=0
$$

This is just the first property of the matrix $\Gamma($ eq. (78)). 
Equation.(78) immediately shows that the second last row of $\underline{N}(r)$ and the second last element of $\mathrm{y}^{(r)}$ vanish identically. Because of equation (79) the contribution to $\mathbb{N}(r)$ from the term $4(z-1)$ in $h_{j}$ vanishes as does the contribution to $\bar{y}^{(r)}$ from the term $I+3(z-1)-$ ln $z$ in $f_{j}$.

The same expressions for $\mathbb{N}(r)$ and $\mathrm{y}^{(r)}(r=0,1,2 \ldots$. . ) can be used when the thermodynamic state is specified by temperature and pressure except that now the last row and last column of $\mathbb{N}(r)$ as well as the last element of $\mathrm{y}^{(\mathrm{r})}$ must be deleted. When entropy and pressure are used as the thermodynamic variables, it is only necessary to replace the last row of $\underline{N}^{(r)}$ with

$$
\begin{aligned}
& \left(\begin{array}{lll}
\sum_{j=1}^{m} a_{k j} s_{j} n_{j} & \frac{S}{R} & s_{T}+\sum_{j=1}^{m} s_{j} h_{j} n_{j}
\end{array}\right) \\
& r=0 \\
& \left(\sum_{j, t=1}^{m} s_{j} n_{j}\left(\underline{\Gamma}^{r}\right)_{j t^{a} k t} \quad 0 \quad \sum_{j, t=1}^{m} s_{j} n_{j}\left(\underline{\Gamma}^{r}\right)_{j t} t_{t}\right) \\
& k=1,2,3 \ldots 2 \\
& r=1,2,3 \ldots .
\end{aligned}
$$

The last element of $y^{(r)}$ must be replaced by

$$
\left.\begin{array}{cc}
\frac{\bar{A} \Delta s}{\bar{R}}+(2-z-\ln Z) \Delta P+\sum_{j=1}^{m} s_{j n} n^{f_{j}} & r=0 \\
\sum_{j, t=1}^{m} s_{j} n_{j}\left(\underline{\Gamma}^{r}\right)_{j t} f_{t} & r=1,2,3 \cdots \cdot
\end{array}\right\}
$$

where the following notation was used:

$$
\begin{gathered}
s_{j} \equiv\left[\frac{\partial\left(\frac{S}{R}\right)}{\partial n_{j}}\right]_{T, P}+2-z-\ln z=\frac{\left(s_{T}^{\circ}\right)_{j}}{R}-\ln n_{j}+\frac{z+1}{Z} \sum_{i=1}^{m} n_{i} \frac{d z}{d a} a_{j} \\
s_{T} \equiv T\left[\frac{\partial\left(\frac{S}{R}\right)}{\partial T}\right]_{P, n_{i}}=\sum_{i=1}^{m} \frac{\left(c_{p}^{O}\right)_{i} n_{i}}{R}+\frac{z+I}{Z} \sum_{i=1}^{m} n_{i} \frac{d z}{d a} \alpha_{T}
\end{gathered}
$$


A calculating scheme has been presented for obtaining equilibrium compositions and thermodynamic properties of plasmas containing many species. This scheme was made compatible with an existing calculating method for ideal gas mixtures by using a perturbation technique for generating a reduced set of working equations.

The method permits the inclusion of both Debye-Huickel and ionization potential lowering effects in the calculation of plasma properties. These two effects are partially compensating.

The equation of state was inverted in order to permit specifying the thermodynamic state in terms of pressure and temperature (or a function of temperature) rather than the usual way of assigning volume and temperature.

The method given also permits the direct calculation of thermodynamic derivatives such as heat capacity and thus elimates the necessity of using the usually less accurate method of numerical differentiation.

Lewis Research Center,

National Aeronautics and Space Administration, Cleveland, Ohio, February 17, 1965. 


\section{APPENDIX - SYMBOIS}

A Helmholtz free energy

$\bar{A} \quad$ total mass of system

$a_{i j} \quad$ stoichiometric coefficients

a $\quad$ Bohr radius

$\mathrm{C}_{\mathrm{p}} \quad$ heat capacity at constant pressure

E energy

$E_{O}^{(i)}$ ground state energy level for $i^{\text {th }}$ isolated species

F Gibbs free energy

H enthalpy

h enthalpy per unit mass

z Planck's constant divided by $2 \pi$

I ionization potential

k Boltzmann's constant

$m_{i} \quad$ mass per particle of species $i$

$\mathrm{N}_{\mathrm{i}}$ number of particles of species $i$

$n_{i} \quad$ number of moles of species $i$

P absolute pressure

p partial pressure

Qint intermal partition function

$Q_{t r} \quad$ translational partition function

$q_{i} \quad$ charge on $i^{\text {th }}$ species

R universal gas constant

S entropy

s entropy per unit mass

T absolute temperature 


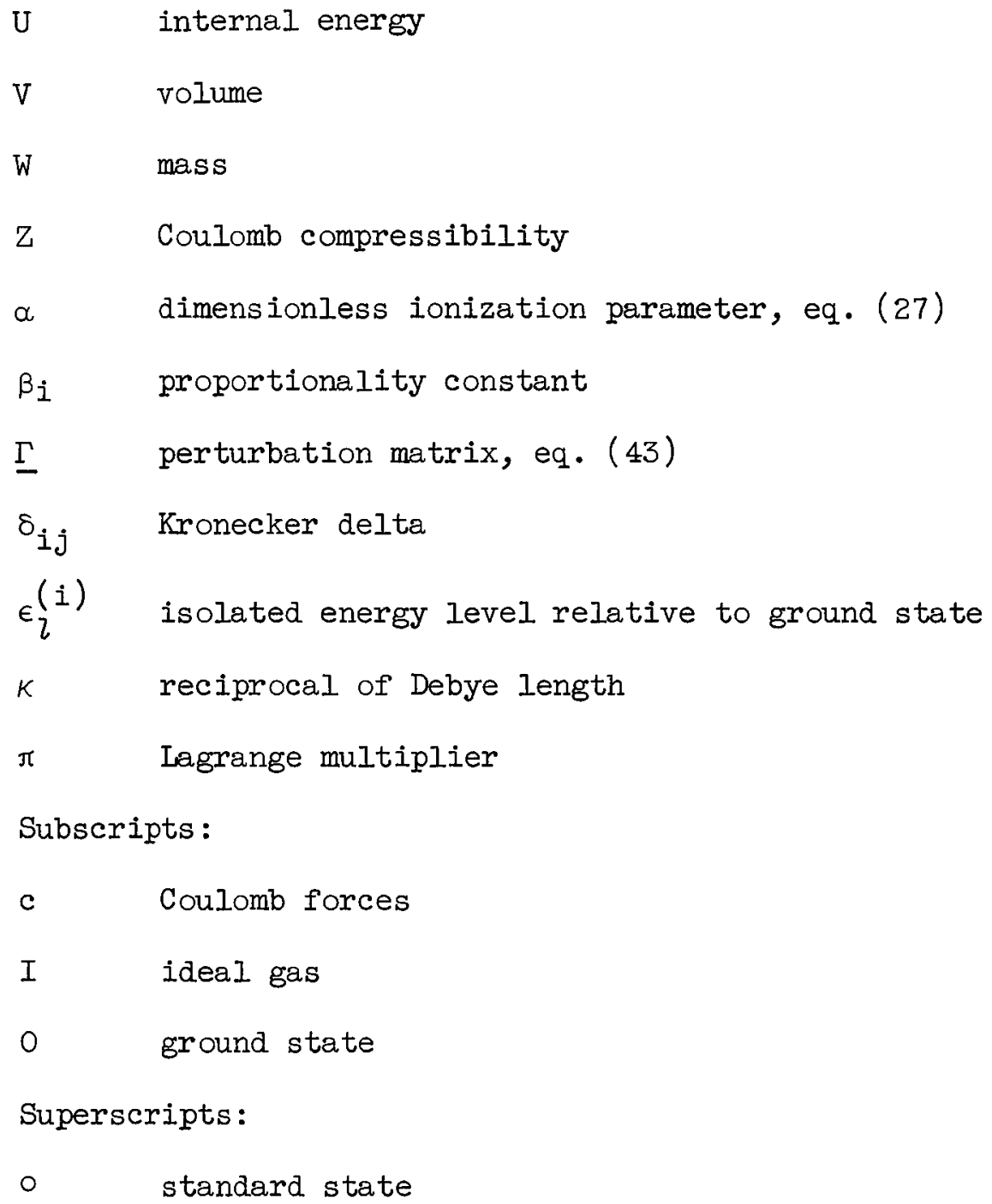


1. Zelezrik, Frank J.; and Gordon, Sanford: An Analytical Investigation of Three General Methods of Calculating Chemical-Equilibrium Compositions. NASA TN D-473, 1960.

2. Ienard, A.: Exact Statistical Mechanics of a One-Dimensional System with Coulomb Forces. J. Math. Phys., vol. 2, 1961, pp. 682-693.

3. Edwards, S. F.; and Ienard, A.: Exact Statistical Mechanics of a OneDimensional System with Coulomb Forces. II. The Method of Functional Integration. J. Math. Phys., vol. 3, 1962, pp. 778-792.

4. Kubin, Robert F.; and Presley, Leroy L.: Thermodynamic Properties and Mollier Chart for Hydrogen from $300^{\circ} \mathrm{K}$ to $20,000^{\circ} \mathrm{K}$. NASA SP-3002, 1964.

5. Hilsenrath, Joseph; Klein, Max; and Woolley, Harold W.: Tables of Thermodynamic Properties of Air Including Dissociation and Ionization from 1,500 $\mathrm{K}$ to $15,000^{\circ} \mathrm{K}$. TR-59-20, Arnold Eng. Dev. Center, Dec. 1959.

6. Rouse, Carl A.: Ionization-Equilibrium Equation of State. Astrophys. J., vol. 134, no. 2, Sept. 1961, pp. 435-446.

7. Rouse, Carl A.: Ionization-Equilibrium Equation of State. II. Mixtures . Astrophys. J., vol. 135, no. 2, Mar. 1962, pp. 599-615.

8. Rosenbaum, Burt M.; and Levitt, Leo: Thermodynamic Properties of Hydrogen from Room Temperature to $100,000^{\circ} \mathrm{K}$. NASA TN D-1107, 1962.

9. Unsöld, A.: Zur Berechnung der Zustandsummen für Atome und Ionen in einem teilweise ionisierten Gas (On the Calculation of Partition Functions for Atoms and Ions in a Partly Ionized Gas). Z. Astrophys., bd. 24, nos. 3-4, 1948, pp. 355-362.

10. Ecker, G.; and Weizel, W.: Zustandsumme und effektive Ionisierungsspannung eines Atoms im Inneren des Plasmas (The Partition Function and Effective Ionization Potential of an Atom in the Interior of the Plasma). Ann. Physik, bd. 17, nos. 2-3, 1956, pp. 126-140.

11. Margenau, H.; and Lewis, M.: Structure of Spectral Lines from Plasmas. Rev. Mod. Phys., vol. 31, no. 3, July 1959, pp. 569-615.

12. Harris, Gilda M.: Attractive Two-Body Interactions in Partially Ionized Plasmas. Phys. Rev., vol. 125, no. 4, Feb. 15, 1962, pp. 1131-1140.

13. Kudrin, L. P.; and Tarasov, Yu. A.: Energy-Level Shifts and the Equation of State of a Plasma. Soviet Phys.-JETP, vol. 16, no. 4, Apr. 1963, pp. $1062-1072$.

14. Shanks, E. Baylis: A Mathematical Treatment of the Problem of Determining the Eigenvalues Associated with a Partition Function of an Atom in the Interior of a Plasma. NASA TN D-111l, 1963. 


\section{ERRATA}

NASA Technical Note D-2806

EQUILIBRTUM COMPUTATIONS FOR MULTICOMPONENT PLASMAS

By Frank J. Zeleznik and Sanford Gordon May 1965

Page 15: Line 8 should read

$\underline{M}_{-1}^{-1}=(\underline{I}+\underline{I})^{-1}$, where $\Lambda=\Gamma$ except for equations (57) and (58). The need for numerically calculating $M_{-1}^{-1}$ would be

Page 15: All $\Gamma^{\prime}$ 's below line 8 should be changed to $\Lambda^{\prime} \mathrm{s}$.

Page 16, equation (67): The $\Gamma^{\prime} \mathrm{s}$ should be changed to $\Lambda$ 's. 